\title{
Certified Clinical Research Associate
}

National Cancer Institute

\section{Source}

National Cancer Institute. Certified Clinical Research Associate. NCI Thesaurus. Code C71296.

A clinical research associate or monitor with related work experience and with certification earned by passing a required program and written examination from the Association of Clinical Research Professionals (ACRP) or equivalent organization. 